\title{
Occasionally Weakly Compatible Mappings
}

\author{
Amit Kumar Govery ${ }^{1, *}$, Mamta Singh ${ }^{2}$ \\ ${ }^{1}$ School of Studies in Mathematics, Vikram University, Ujjain - 456010 (M.P.), India \\ ${ }^{2}$ Department of Mathematical Science and Computer Application, Bundelkhand University, Jhansi (U.P.), India \\ *Corresponding author: amitgovery@gmail.com
}

Received June 08, 2015; Revised July 15, 2015; Accepted July 24, 2015

\begin{abstract}
In this paper, the concept of compatible maps of type (A) and occasionally weakly compatible maps in fuzzy metric space have been applied to prove common fixed point theorem. A fixed point theorem for six self maps has been established using the concept of compatible maps of type (A) and occasionally weakly compatible maps, which generalizes the result of Cho [16].
\end{abstract}

Keywords: common fixed points, fuzzy metric space, compatible maps, compatible maps of type (A) and occasionally weakly compatible maps

Cite This Article: Amit Kumar Govery, and Mamta Singh, "Occasionally Weakly Compatible Mappings." Turkish Journal of Analysis and Number Theory, vol. 3, no. 3 (2015): 78-82. doi: 10.12691/tjant-3-3-2.

\section{Introduction}

The concept of Fuzzy sets was initially investigated by Zadeh [13] as a new way to represent vagueness in everyday life. Subsequently, it was developed by many authors and used in various fields. To use this concept in Topology and Analysis, several researchers have defined Fuzzy metric space in various ways. In this paper we deal with the Fuzzy metric space defined by Kramosil and Michalek [11] and modified by George and Veeramani [20]. Recently, Grebiec [1] has proved fixed point results for Fuzzy metric space. In the sequel, Singh and Chauhan [12] introduced the concept of compatible mappings of Fuzzy metric space and proved the common fixed point theorem. Jungck et. al. [2] introduced the concept of compatible maps of type (A) in metric space and proved fixed point theorems. Using the concept of compatible maps of type (A), Jain et. al. [18] proved a fixed point theorem for six self maps in a fuzzy metric space. Singh et. al. $[7,8]$ proved fixed point theorems in a fuzzy metric space. Recently in 2012, Jain et. al. [4,5] and Sharma et. al. [6] proved various fixed point theorems using the concepts of semi-compatible mappings, property (E.A.) and absorbing mappings. The concept of occasionally weakly compatible mappings in metric spaces is introduced by AlThagafi and Shahzad [14] which is most general among all the commutativity concepts. Recently, Khan and Sumitra [15] extended the notion of occasionally weakly compatible maps to fuzzy metric space.

In this paper, a fixed point theorem for six self maps has been established using the concept of compatible maps of type (A) occasionally weakly compatible mappings, which generalizes the result of Cho [16].

For the sake of completeness, we recall some definitions and known results in Fuzzy metric space.

\section{Definitions, lemmas, Remarks, Propositions}

Definition 2.1. [10] A binary operation * : $[0,1] \times[0,1] \rightarrow[0,1]$ is called a $t$-norm if $([0,1], *)$ is an abelian topological monoid with unit 1 such that $a * b \leq c * d$. Whenever $a \leq c$ and $b \leq d$ for $a, b, c, d$. $[0,1]$.

Examples of $t$-norms are:

$$
a * b=a b \text { and } a * b=\min \{a, b\} .
$$

Definition 2.2. [10] The 3-tuple $(X, M, *)$ is said to be a Fuzzy metric space if $\mathrm{X}$ is an arbitrary set, $*$ is a continuous t-norm and $\mathrm{M}$ is a Fuzzy set in $x^{2} \times[0, \infty)$ satisfying the following conditions:

For all $x, y, z \in X$ and $s, t>0$

(FM-1) $M(x, y, 0)=0$,

(FM-2) $M(x, y, t)=1$ for all $t>0$ iff $x=y$,

(FM-3) $M(x, y, t)=M(y, x, t)$,

$(\mathrm{FM}-4) M(x, y, t) * M(y, z, s)=M(x, z, t+s)$,

$(\mathrm{FM}-5) M(x, y,)^{-}:[0, \infty) \rightarrow[0,1]$ is left

continuous,

(FM-6) $\lim _{n \rightarrow \infty} M(x, y, t)=1$

Note that $M(x, y, t)$ can be considered as the degree of nearness between $\mathrm{x}$ and $\mathrm{y}$ with respect to t. We identify $x=y$ with $M(x, y, t)=1$ for all $t>0$. The following example shows that every metric space induces a Fuzzy metric space.

Example 2.1. [10] Let $(X, d)$ be a metric space. Define $a * b=\min \{a, b\}$ and $M(x, y, t)=\frac{t}{t+d(x, y)}$ for all $x, y \in X$ and $t>0$. Then $(X, M, *)$ is a Fuzzy metric space. It is called the Fuzzy metric space induced by $d$. 
Definition 2.3. [10] A sequence $\left\{x_{n}\right\}$ in a Fuzzy metric space $(X, M, *)$ is said to be a Cauchy sequence if and only if for each $\varepsilon>0, \mathrm{t}>0$, there exists $n_{0} \in N$ such that $\mathrm{M}\left(\mathrm{X}_{\mathrm{n}}, \mathrm{X}_{\mathrm{m}}, \mathrm{t}\right)>1-\varepsilon$ for all $\mathrm{n}, \mathrm{m} \geq \mathrm{n}_{0}$.

The sequence $\left\{X_{n}\right\}$ is said to converge to a point $X$ in $X$ if and only if for each $\varepsilon>0, \mathrm{t}>0$ there exists $\mathrm{n}_{0} \in \mathrm{N}$ such that $M\left(x_{n}, x, t\right)>1-\varepsilon$ for all $n, m \geq n_{0}$.

A Fuzzy metric space $(X, M, *)$ is said to be complete if every Cauchy sequence in it converges to a point in it.

Definition 2.4. [12] Self mappings $A$ and $S$ of a Fuzzy metric space $(X, M, *)$ are said to be compatible if and only if $M\left(A S x_{n}, S A x_{n}, t\right) \rightarrow 1$ for all $t>0$, whenever $\left\{x_{n}\right\}$ is a sequence in $X$ such that $S x n, A x n \rightarrow p$ for some $p$ in $X$ as $n \rightarrow \infty$.

Definition 2.5. [18] Self maps $A$ and $S$ of a Fuzzy metric space $(X, M, *)$ are said to be compatible maps of type $(A)$ if $M\left(A S x_{n}, S S x_{n}, t\right) \rightarrow 1$ and $M\left(S A x_{n}, A A x_{n}, t\right) \rightarrow 1$ for all $t>0$, whenever $\left\{x_{n}\right\}$ is a sequence in $X$ such that $S x_{n}, A x_{n} \rightarrow p$ for some $p$ in $X$ as $n \rightarrow \infty$.

Definition 2.6. [15] Two maps $A$ and $S$ from a Fuzzy metric space $(X, M, *)$ into itself are said to be Occasionally weakly compatible (owc) if and only if there is a point $x \in X$, which is coincidence point of $A$ and $S$ at which $A$ and $S$ commute.

Remark 2.1. [18] The concept of compatible maps of type $(A)$ and occasionally weakly compatibility is more general than the concept of compatible maps in a Fuzzy metric space.

Proposition 2.1. [18] In a Fuzzy metric space $(X, M, *)$ limit of a sequence is unique.

Lemma 2.1. [1] Let $(X, M, *)$ be a fuzzy metric space. Then for all $x, y \in X, M(x, y,$.$) is a non-decreasing$ function.

Lemma 2.2. [16] Let $(X, M, *)$ be a fuzzy metric space. If there exists $k \in(0,1)$ such that for all $x, y \in$ $X, M(x, y, k t) \geq M(x, y, t) \forall t>0$,

then $x=y$.

Lemma 2.3. [18] Let $\left\{x_{n}\right\}$ be a sequence in a fuzzy metric space $(X, M, *)$. If there exists a number $k \in(0,1)$ such that $M\left(x_{n+2}, x_{n+1}, k t\right) \geq M\left(x_{n+1}, x_{n}, t\right) \forall t>0$ and $n \in N$. Then $\left\{x_{n}\right\}$ is a Cauchy sequence in $X$.

Proposition 2.2. [18] Let $A$ and $S$ be concept of compatible maps of type $(A)$ of a complete fuzzy metric space $(X, M, *)$ with continuous t-norm * defined by $a * b=\min \{a, b\}$ for all $a, b \in[0,1]$ and $S u=T u$ for some $u$ in $X$. Then STu $=T S u=S S u=T T u$.

Lemma 2.4. [3] The only $t$-norm * satisfying $r * r \geq r$ for all $r \in[0,1]$ is the minimum $t$-norm, that is $a * b=$ $\min \{a, b\}$ for all $a, b \in[0,1]$.

\section{Main Result}

Theorem 3.1. Let $(X, M, *)$ be a complete fuzzy metric space and let $A, B, S, T, P$ and $Q$ be mappings from $X$ into itself such that the following conditions are satisfied:

(a) $P(X) \subset S T(X), Q(X) \subset A B(X)$;

(b) $A B=B A, S T=T S, P B=B P, Q T=T Q$;

(c) either $P$ or $A B$ is continuous; (d) $(P, A B)$ is compatible maps of type $(A)$ and $(Q, S T)$ is occasionally weakly compatible ;

(e) there exists $q \in(0,1)$ such that for every $x, y \in X$ and $t>0$

$$
\begin{aligned}
M(P x, Q y, q t) \geq & M\left(A B x, S T y,{ }^{*} M(P x, A B x, t) v\right. \\
& * M(Q y, S T y, t * M(P x, S T y, t) .
\end{aligned}
$$

Then $A, B, S, T, P$ and $Q$ have a unique common fixed point in $X$.

Proof: Let $x_{0} \in \mathrm{X}$. From (a) there exist $x_{1}, x_{2} \in X$ such that $P x_{0}=S T x_{1}$ and $x_{1}=A B x_{2}$.

Inductively, we can construct sequences $\left\{x_{n}\right\}$ and $\left\{y_{n}\right\}$ in $X$ such that $P x_{2 n-2}=S T x_{2 n-1}=y_{2 n-1}$ and $Q x_{2 n-1}=A B x_{2 n}=y_{2 n}$ for $n=1,2,3, \ldots$

Step 1. Put $x=x_{2 n}$ and $y=x_{2 n+1}$ in (e), we get

$$
\begin{aligned}
& M\left(P x_{2 n}, Q x_{2 n+1}, q t\right) \\
\geq & M\left(A B x_{2 n}, S T x_{2 n+1}, t\right) * M\left(P x_{2 n}, A B x_{2 n}, t\right) \\
& * M\left(Q x_{2 n+1}, S T x_{2 n+1}, t\right) * M\left(P x_{2 n}, S T x_{2 n+1}, t\right) . \\
= & M\left(y_{2 n}, y_{2 n+1}, t\right) * M\left(y_{2 n+1}, y_{2 n}, t\right) \\
& * M\left(y_{2 n+2}, y_{2 n+1}, t\right) * M\left(y_{2 n+1}, y_{2 n+1}, t\right) \\
\geq & M\left(y_{2 n}, y_{2 n+1}, t\right) * M\left(y_{2 n+1}, y_{2 n+2}, t\right) .
\end{aligned}
$$

From lemma 2.1 and 2.2, we have

$$
M\left(y_{2 n+1}, y_{2 n+2}, q t\right) \geq M\left(y_{2 n}, y_{2 n+11}, t\right) .
$$

Similarly, we have

$$
M\left(y_{2 n+2}, y_{2 n+3}, q t\right) \geq M\left(y_{2 n+1}, y_{2 n+2}, t\right) .
$$

Thus, we have

$$
\begin{gathered}
M\left(y_{n+1}, y_{n+2}, q t\right) \geq M\left(y_{n}, y_{n+1}, t\right) \\
\text { for } n=1,2, \ldots \\
M\left(y_{n}, y_{n+1}, t\right) \geq M\left(y_{n}, y_{n+1}, t / q\right) \\
\geq M\left(y_{n-2}, y_{n-1}, t / q 2\right) \\
\ldots \ldots \ldots \ldots \\
\geq M\left(y_{1}, y_{2}, t / q^{n}\right) \rightarrow 1 \text { as } n \rightarrow \infty,
\end{gathered}
$$

and hence $M\left(y_{n}, y_{n+1}, t\right) \rightarrow 1$ as $n \rightarrow \infty$ for any $t>0$.

For each $\varepsilon>0$ and $t>0$, we can choose $n_{0} \in N$ such that

$$
M\left(y_{n}, y_{n+1}, t\right)>1-\varepsilon \text { for all } n>n_{0} .
$$

For $m, n \in N$, we suppose $m \geq n$. Then we have

$$
\begin{aligned}
& M\left(y_{n}, y_{m}, t\right) \geq M\left(y_{n}, y_{n+1}, t / m-n\right) \\
& * M\left(y_{n+1}, y_{n+2}, t / m-n\right. \\
& * \ldots * M\left(y_{m-1}, y_{m}, t / m-n\right. \\
& \geq(1-\varepsilon) *(1-\varepsilon) * \ldots *(1-\varepsilon) \\
& M\left(y_{n}, y_{m}, t\right) \geq(1-\varepsilon)
\end{aligned}
$$

and hence $\left\{y_{n}\right\}$ is a Cauchy sequence in $X$.

Since $(X, M, *)$ is complete, $\left\{y_{n}\right\}$ converges to some point $z \in X$. Also its subsequence's converges to the same point i.e. $Z \in X$

$$
\begin{gathered}
\text { i.e., }\left\{Q x_{2 n+1}\right\} \rightarrow z \text { and }\left\{S T x_{2 n+1}\right\} \rightarrow z \\
\left\{P x_{2 n}\right\} \rightarrow z \text { and }\left\{A B x_{2 n}\right\} \rightarrow z
\end{gathered}
$$


Case I. Suppose $A B$ is continuous.

Since $A B$ is continuous, we have

$$
(A B)^{2} x_{2 n} \rightarrow A B z \text { and } A B P x_{2 n} \rightarrow A B z .
$$

As $(P, A B)$ is compatible pair of type $(A)$, we have $P A B x_{2 n} \rightarrow A B z$.

Step 2. Put $x=A B x_{2 n}$ and $y=x_{2 n+1}$ in (e), we get

$$
\begin{aligned}
& M\left(P A B x_{2 n}, Q x_{2 n+1}, q t\right) \\
\geq & M\left(A B A B x_{2 n}, S T x_{2 n+1}, t\right) * M\left(P A B x_{2 n}, A B A B x_{2 n}, t\right) \\
& * M\left(Q x_{2 n+1}, S T x_{2 n+1}, t\right) * M\left(P A B x_{2 n}, S T x_{2 n+1}, t\right) .
\end{aligned}
$$

Taking $\rightarrow \infty$, we get

$$
\begin{gathered}
M(A B z, z, q t) \geq M(A B z, z, t) * M(A B z, A B z, \\
\quad * M(z, z, t) * M(A B z, z, t) \\
\geq M(A B z, z, t) * M(A B z, z, t) \\
\text { i.e. } M(A B z, z, q t) \geq M(A B z, z, t) .
\end{gathered}
$$

Therefore, by using lemma 2.2, we get

$$
A B z=z \text {. }
$$

Step 3. Put $x=z$ and $y=x 2 n+1$ in (e), we have

$$
\begin{aligned}
& M\left(P z, Q x_{2 n+1}, q t\right. \\
\geq & M\left(A B z, S T x_{2 n+1}, t\right) * M(P z, A B z, t) \\
& * M\left(Q x_{2 n+1}, S T x_{2 n+1}, t\right) * M\left(P z, S T x_{2 n+1}, t\right) .
\end{aligned}
$$

Taking $n \rightarrow \infty$ and using equation (1), we get

$$
\begin{aligned}
M(P z, z, q t) \geq & M(z, z, t) * M(P z, z, t) \\
& * M(z, z, t) * M(P z, z, t) \\
\geq & M(P z, z, t) * M(P z, z, t)
\end{aligned}
$$

i.e. $M(P z, z, q t) \geq M(P z, z, t)$.

Therefore, by using lemma 2.2, we get

$$
P z=z \text {. Therefore, } A B z=P z=z \text {. }
$$

Step 4. Putting $x=B z$ and $y=x_{2 n+1}$ in condition (e), we get

$$
\begin{aligned}
& M\left(P B z, Q x_{2 n+1}, q t\right) \\
\geq & M\left(A B B z, S T x_{2 n+1}, t\right) * M(P B z, A B B z, t) \\
& * M\left(Q x_{2 n+1}, S T x_{2 n+1}, t\right) * M\left(P B z, S T x_{2 n+1}, t\right) .
\end{aligned}
$$

As $B P=P B, A B=B A$, so we have

$$
\begin{aligned}
& P(B z)=B(P z)=B z a n d(A B)(B z) \\
& =(B A)(B z)=B(A B z)=B z .
\end{aligned}
$$

Taking $n \rightarrow \infty$ and using (1), we get

$M(B z, z, q t)$

$\geq M(B z, z, t) * M(B z, B z, t) * M(z, z, t) * M(B z, z, t)$

$\geq M(B z, z, t) * M(B z, z, t)$

i.e. $M(B z, z, q t) \geq M(B z, z, t)$.

Therefore, by using lemma 2.2, we get

$B z=z$ and also we have $A B z=Z \Rightarrow A z=Z$.

Therefore,

$$
A z=B z=P z=z
$$

Step 5. As $P(X) \subset S T(X)$, there exists $u \in X$ such that $z=P z=S T u$.

Putting $x=x_{2 n}$ and $y=u$ in (e), we get

$$
\begin{aligned}
& M\left(P x_{2 n}, Q u, q t\right) \\
\geq & M\left(A B x_{2 n}, S T u, t\right) * M\left(P x_{2 n}, A B x_{2 n}, t\right) \\
& * M(Q u, S T u, t) * M(P x 2 n, S T u, t) .
\end{aligned}
$$

Taking $n \rightarrow \infty$ and using (1) and (2), we get

$$
\begin{aligned}
M(z, Q u, q t) \geq & M(z, z, t) * M(z, z, t) \\
& * M(Q u, z, t) * M(z, z, t) * M(z, z, t) \\
\geq & M(Q u, z, t)
\end{aligned}
$$

i.e. $M(z, Q u, q t) \geq M(z, Q u, t)$.

Therefore, by using lemma 2.2, we get $Q u=z$. Hence $S T u=z=Q u$. Since $(Q, S T)$ is occasionally weakly compatible, therefore, by proposition (2.2), we have

$$
Q S T u=S T Q u \text {. Thus } Q z=S T z \text {. }
$$

Step 6. Putting $x=x_{2 n}$ and $y=z$ in (e), we get

$$
\begin{aligned}
& M\left(P x_{2 n}, Q z, q t\right) \\
& \geq M\left(A B x_{2 n}, S T z, t\right) * M\left(P x_{2 n}, A B x_{2 n}, t\right) \\
& \quad * M(Q z, S T z, t) * M\left(P x_{2 n}, S T z, t\right) .
\end{aligned}
$$

Taking $n \rightarrow \infty$ and using (2) and step 5, we get

$$
\begin{aligned}
M(z, Q z, q t) \geq & M(z, Q z, t) * M(z, z, t) \\
& * M(Q z, Q z, t) * M(z, Q z, t) \\
\geq & M(z, Q z, t) * M(z, Q z, t)
\end{aligned}
$$

i.e. $M(z, Q z, q t) \geq M(z, Q z, t)$.

Therefore, by using lemma 2.2 , we get

$$
\mathrm{Qz}=\mathrm{z} \text {. }
$$

Step 7. Putting $x=x_{2 n}$ and $y=T z$ in (e), we get

$$
\begin{aligned}
& M\left(P x_{2 n}, Q T z, q t\right) \\
\geq & M\left(A B x_{2 n}, S T T z, t\right) * M\left(P x_{2 n}, A B x_{2 n}, t\right) \\
& * M(Q T z, S T T z, t) * M\left(P x_{2 n}, S T T z, t\right) .
\end{aligned}
$$

As $Q T=T Q$ and $S T=T S$, we have

$$
\begin{aligned}
& Q T z=T Q z=T z \\
& \text { and } S T(T z)=T(S T z)=T Q z=T z .
\end{aligned}
$$

Taking $n \rightarrow \infty$, we get

$$
\begin{aligned}
M(z, T z, q t) \geq & M(z, T z, t) * M(z, z, t) \\
& * M(T z, T z, t) * M(z, T z, t) \\
\geq & M(z, T z, t) * M(z, T z, t)
\end{aligned}
$$

i.e. $M(z, T z, q t) \geq M(z, T z, t)$

Therefore, by using lemma 2.2, we get

$T z=z$. Now $S T z=T z=z$ implies $S z=z$.

Hence

$$
S z=T z=Q z=z .
$$

Combining (4) and (5), we get

$$
A z=B z=P z=Q z=T z=S z=z .
$$


Hence, $z$ is the common fixed point of $A, B, S, T, P$ and $Q$.

Case II. Suppose $P$ is continuous.

As $P$ is continuous,

$$
P^{2} x_{2 n} \rightarrow P z \text { and } P(A B) x_{2 n} \rightarrow P z \text {. }
$$

As $(P, A B)$ is compatible pair of type $(A)$, $(A B) P x_{2 n} \rightarrow P z$.

Step 8. Putting $x=P x_{2 n}$ and $y=x_{2 n+1}$ in condition (e), we have

$$
\begin{aligned}
& M\left(P P x_{2 n}, Q x_{2 n+1}, q t\right) \\
& \geq M\left(A B P x_{2 n}, S T x_{2 n+1}, t\right) * M\left(P P x_{2 n}, A B P x_{2 n}, t\right) \\
& \quad * M\left(Q x_{2 n+1}, S T x_{2 n+1}, t\right) * M\left(P P x_{2 n}, S T x_{2 n+1}, t\right) .
\end{aligned}
$$

Taking $n \rightarrow \infty$, we get

$$
\begin{aligned}
M(P z, z, q t) \geq & M(P z, z, t) * M(P z, P z, t) \\
& * M(z, z, t) * M(P z, z, t) \\
\geq & M(P z, z, t) * M(P z, z, t)
\end{aligned}
$$

i.e. $M(P z, z, q t) \geq M(P z, z, t)$.

Therefore by using lemma 2.2 , we have

$$
P z=z \text {. }
$$

Further, using steps 5,6,7, we get

$$
Q z=S T z=S z=T z=z .
$$

Step 9. As $Q(X) \subset A B(X)$, there exists $w \in X$ such that $z=Q z=A B w$

Put $x=w$ and $y=x_{2 n+1}$ in (e), we get

$$
\begin{aligned}
& M\left(P w, Q x_{2 n+1}, q t\right) \\
\geq & M\left(A B w, S T x_{2 n+1}, t\right) * M(P w, A B w, t) \\
& * M\left(Q x_{2 n+1}, S T x_{2 n+1}, t\right) * M\left(P w, S T x_{2 n+1}, t\right) .
\end{aligned}
$$

Taking $n \rightarrow \infty$, we get

$$
\begin{aligned}
M(P w, z, q t) \geq & M(z, z, t) * M(P w, z, t) \\
& * M(z, z, t) * M(P w, z, t) \\
\geq & M(P w, z, t) * M(P w, z, t)
\end{aligned}
$$

i.e. $M(P w, z, q t) \geq M(P w, z, t)$.

Therefore, by using lemma 2.2, we get

$$
P w=z \text {. Therefore, } A B w=z=P w .
$$

As $(P, A B)$ is compatible pair of type $(A)$, then by proposition (2.2), we have

$$
P z=A B Z \text {. }
$$

Also, from step 4, we get $B z=z$.

$$
A z=B z=P z=z .
$$

Further, using steps $5,6,7$, we get

$$
Q z=S T z=S z=T z=z
$$

i.e. $Z$ is the common fixed point of the six maps $A, B, S, T, P$ and $Q$ in this case also.

Uniqueness: Let $u$ be another common fixed point of $A, B, S, T, P$ and $Q$.

Then $A u=B u=P u=Q u=S u=T u=u$. Put $x=z$ and $y=u$ in (e), we get

$$
\begin{array}{r}
M(P z, Q u, q t) \geq M(A B z, S T u, t) * M(P z, A B z, t) \\
* M(Q u, S T u, t) * M(P z, S T u, t) .
\end{array}
$$

Taking $n \rightarrow \infty$, we get

$$
\begin{aligned}
M(z, u, q t) \geq & M(z, u, t) * M(z, z, t) \\
& * M(u, u, t) * M(z, u, t) \\
\geq & M(z, u, t) * M(z, u, t)
\end{aligned}
$$

i.e. $M(z, u, q t) \geq M(z, u, t)$.

Therefore by using lemma (2.2), we get $z=u$.

Therefore $z$ is the unique common fixed point of self maps $A, B, S, T, P$ and $Q$.

Remark 3.1. If we take $B=T=I$, the identity map on $X$ in theorem 3.1 , then condition $(b)$ is satisfied trivially and we get

Corollary 3.1. Let $(X, M, *)$ be a complete fuzzy metric space and let $A, S, P$ and $Q$ be mappings from $X$ into itself such that the following conditions are satisfied:

(a) $P(X) \subset S(X), Q(X) \subset A(X)$;

(b) either $A$ or $P$ is continuous;

(c) $(P, A)$ is compatible maps of type $(A)$ and $(Q, S)$ is occasionally weakly compatible;

(d) there exists $q \in(0,1)$ such that for every $x, y \in X$ and $t>0$

$$
\begin{aligned}
M(P x, Q y, q t) \geq & M(A x, S y, t) * M(P x, A x, t) \\
& * M(Q y, S y, t) * M(P x, S y, t) .
\end{aligned}
$$

Then $A, S, P$ and $Q$ have a unique common fixed point in $X$.

Remark 3.2. In view of remark 3.1 , corollary 3.1 is a generalization of the result of Cho [16] in the sense that condition of compatibility of the pairs of self maps has been restricted to compatibility of type $(A)$ occasionally weakly compatible and only one map of the first pair is needed to be continuous.

\section{Acknowledgement}

Authors are thankful to the referee for his valuable comments.

\section{References}

[1] George and P. Veeramani, On some results in Fuzzy metric spaces, Fuzzy Sets and Systems 64 (1994), 395-399.

[2] Jain and B. Singh, A fixed point theorem for compatible mappings of type (A) in fuzzy metric space, Acta Ciencia Indica, Vol. XXXIII M, No. 2 (2007), 339-346.

[3] Jain, M. Sharma and B. Singh, Fixed point theorem using compatibility of type ( $\beta$ ) in Fuzzy metric space, Chh. J. Sci. \& Tech., Vol. 3 \& 4, (2006- 2007), 53-62.

[4] Jain, V.H. Badshah, S.K. Prasad, Fixed Point Theorem in Fuzzy Metric Space for Semi-Compatible Mappings, Int. J. Res. Rev. Appl. Sci. 12 (2012), 523-526.

[5] Jain, V.H. Badshah, S.K. Prasad, The Property (E.A.) and The Fixed Point Theorem in Fuzzy Metric, Int. J. Res. Rev. Appl. Sci. 12 (2012), 527-530.

[6] Sharma, A. Jain, S. Chaudhary, A note on absorbing mappings and fixed point theorems in fuzzy metric space, Int. J. Theoretical Appl. Sci. 4 (2012), 52-57.

[7] Singh, A. Jain, A.K. Govery, Compatibility of type $(\beta)$ and fixed point theorem in Fuzzy metric space, Appl. Math. Sci. 5 (2011), 517-528.

[8] Singh, A. Jain, A.K. Govery, Compatibility of type (A) and fixed point theorem in Fuzzy metric space,Int. J. Contemp. Math. Sci. 6 (2011), 1007-1018. 
[9] Singh and M.S. Chouhan, Common fixed points of compatible maps in Fuzzy metric spaces, Fuzzy sets and systems, 115 (2000), 471-475.

[10] E.P. Klement, R. Mesiar and E. Pap, Triangular Norms, Kluwer Academic Publishers.

[11] G. Jungck, P.P. Murthy and Y.J. Cho, Compatible mappings of type (A) and common fixed points, Math. Japonica, 38 (1993), 381-390.

[12] I.Kramosil and J. Michalek, Fuzzy metric and statistical metric spaces, Kybernetica 11(1975), 336-344.

[13] L. A. Zadeh, Fuzzy sets, Inform and control 89 (1965), 338-353.

[14] M.A. Al-Thagafi, N.A. Shahzad, A note on occasionally weakly compatible maps, Int. J. Math. anal. 3(2009), 55-58.
[15] M.A. Khan, Sumitra, Common fixed point theorems for occasionally weakly compatible maps in fuzzy metric spaces, Far East J. Math. Sci., 9 (2008), 285-293.

[16] S.H., Cho, On common fixed point theorems in fuzzy metric spaces, J. Appl. Math. \& Computing Vol. 20 (2006), No. 1-2, 523-533.

[17] S.N. Mishra, N. Mishra and S.L. Singh, Common fixed point of maps in fuzzy metric space, Int. J. Math. Math. Sci. 17(1994), 253258.

[18] M. Grebiec, Fixed points in Fuzzy metric space, Fuzzy sets and systems, 27(1998), 385-389.

[19] Y.J. Cho, Fixed point in Fuzzy metric space, J. Fuzzy Math. 5(1997), 949-962.

[20] Y.J. Cho, H.K. Pathak, S.M. Kang and J.S. Jung, Common fixed points of compatible mappings of type ( $\beta$ ) on fuzzy metric spaces, Fuzzy sets and systems, 93 (1998), 99-111. 